\title{
Dual-modal ablation treatment for refractory radiation proctitis
}

\author{
Shajan Peter ${ }^{1} \cdot$ Kevin P. Cowley ${ }^{2} \cdot$ C. Mel Wilcox ${ }^{1} \cdot$ Klaus Mönkemüller $^{1}$
}

Accepted: 29 July 2015 / Published online: 15 August 2015

(C) Springer-Verlag Berlin Heidelberg 2015

\section{Dear Editor:}

A 77-year-old male with recent history of radiation therapy for prostate cancer was referred with symptoms of fresh bleeding per rectum. Radiation proctitis (RP) was previously diagnosed on endoscopy at an outside facility. He had undergone three endoscopic ablation treatments with argon plasma coagulation (APC) but lower GI bleeding persisted, requiring multiple blood transfusions. On flexible sigmoidoscopy, multiple bleeding telangiectatic lesions with erythema and friability extending to $10 \mathrm{~cm}$ from the anal verge were noted, consistent with moderate grade radiation proctitis. Radiofrequency ablation (RFA) was offered as a rescue treatment. RFA was performed using the HALO 90 system (Covidien, Sunnyvale, CA), and the scope was rotated circumferentially to target all areas. Energy was delivered at settings of $12-15 \mathrm{~J} / \mathrm{cm}^{2}$ and power density of $40 \mathrm{~W} / \mathrm{cm}^{2}$. At least two ablation sets were applied to each area. Post ablation, the coagulum was not scraped to promote hemostasis. The patient underwent a total of three RFA sessions at 4- to 6-week intervals. His symptoms markedly improved with treatment sessions. On repeat endoscopy, the patient was found to have few spotty telangiectasias that were ablated with APC, $30 \mathrm{~W}$, pulsed at $2 \mathrm{~s}$ (Erbe, Tübingen,

Kevin P. Cowley

kcowley@uabmc.edu

1 Division of Gastroenterology and Hepatology, Basil Hirschowitz Endoscopic Center of Excellence, University of Alabama at Birmingham, Birmingham, AL, USA

2 Tinsley Harrison Internal Medicine Program, University of Alabama at Birmingham, Birmingham, AL, USA
Germany). On close follow-up, he was asymptomatic and hemoglobin levels were stable from prior.

Rectal bleeding can occur from chronic radiation proctitis in up to $20 \%$ of patients as a late complication following radiation therapy for pelvic malignancy. Ablation of the abnormal microvessels with subsequent re-epithelization is beneficial, and APC is currently offered as treatment of choice. However, in refractory cases, endoscopic RFA has recently been proposed as an alternative treatment for RP.

RFA confers additional advantages over other modalities such as APC, as illustrated as follows. Firstly, RFA allows simultaneous treatment of broader areas of targeted tissue compared to APC. Also, RFA delivers a consistent amount of energy to the surface causing uniform coagulation of vessels achieving adequate hemostasis. Finally, by the tightly spaced bipolar array, RFA has limited energy penetration, which avoids energy dissipation and further injury to deeper tissue.

The technical challenge was to maintain an optimal apposition to the targeted tissue applying torque and rotation of the scope with the electrode attached over the tip in a clockwise fashion, such that circumferential ablation to all areas was feasible. Targeting lesions close to the dentate line may be better using APC. This unique case exhibits a "dual" approach to achieve complete resolution of symptoms and bleeding related to chronic RP. In this proposed dual approach, RFA serves to deliver an initial ablative "hit" by significantly decreasing the burden of lesions, and APC is used to subsequently target the remaining point lesions. We believe that this combined approach also will prevent ulceration and long-term structuring compared to a single-mode RFA treatment. In conclusion, dual-modal ablation offers an ideal approach to management of RP, especially in refractory cases. 